\title{
Solar System Wave Function
}

\author{
Abolfazl Soltani \\ Department of Physics, University of Birjand, Birjand, Iran
}

Email: Soltani.a.physics@gmail.com

\begin{abstract}
Pluto, Ceres and all planets of Solar system except Neptune, with a high approximation, follow a rule called Titius-Bode rule or Bode rule, which can by no means be considered as a stochastic event. This rule shows that the distance of planets from the sun in Solar system is regulated. Here, I prove that the existence of a standing and cosine wave packet, with the wavelength $\lambda=0.6 \mathrm{AU}$ ( $A U$ represents the distance of Earth from the Sun) and the phase constant $\varnothing=\frac{\pi}{6}$, in solar system is the reason for Bode rule. And moreover, I prove that this huge wave packet belongs to the sun. Bode rule does not predict the distance of Neptune from the Sun but, this article is able to give us the distance of Neptune. Based on this article, quantum mechanics and physics will enter a new stage.
\end{abstract}

Keywords: Solar system, Titius-Bode rule, Quantum mechanics

\section{Introduction}

The planets of solar system move around the sun in elliptical orbits such that the sun is in one of the focal points of these ellipses. These ellipses are very close to the circle, and in fact the orbits of the planets of solar system are concentric circles. Pluto and Ceres and all planets of Solar system except Neptune, with a high approximation follow a rule known as Bode rule or Titius-Bode rule. According to this rule, the distance of each planet from the Sun is equal to $a=0.4 A U+0.3 A U \times 2^{n}$, where $0.4 A U$ is the distance of Mercury from the Sun (or more precisely the length of the semi-major axis of Mercury's orbit) and $n=0,1,2,3, \ldots$. [1]. Table. 1 shows the high accuracy of the Bode rule. If this rule was only true for three or four planets, then we could call it a coincidence, but when it is true for seven planets, plus Ceres and Pluto, there is definitely a reason for it. It was historically based on this rule that Ceres was discovered in 1801 [1]. In this article, I will find the reason for the existence of the Bode rule. In fact, I will prove that the presence of a cosine and standing wave packet in solar system is the reason for existence of Bode rule. and, I will prove that this wave packet belongs to the sun. The Bode rule does not predict the position of Neptune but my wave theory, which believes in the presence of a cosine and standing wave in solar system, can give us the position of Neptune. 


\begin{tabular}{|c|c|c|c|}
\hline Planet & $\begin{array}{c}\text { T-B rule } \\
\text { distance }(\mathrm{AU})\end{array}$ & $\begin{array}{c}\text { Semi-major } \\
\text { axis (AU) }\end{array}$ & $\begin{array}{c}\text { Deviation from } \\
\text { prediction }\end{array}$ \\
\hline Mercury & 0.4 & 0.39 & $-2.5 \%$ \\
\hline Venus & 0.7 & 0.72 & $+2.8 \%$ \\
\hline Earth & 1.0 & 1.00 & $0.00 \%$ \\
\hline Mars & 1.6 & 1.52 & $-4.77 \%$ \\
\hline Ceres & 2.8 & 2.77 & $-1.16 \%$ \\
\hline Jupiter & 5.2 & 5.20 & $+0.00 \%$ \\
\hline Saturn & 10.0 & 9.58 & $-4.45 \%$ \\
\hline Uranus & 19.6 & 19.20 & $-1.95 \%$ \\
\hline Pluto & 38.8 & 39.48 & $+1.05 \%$ \\
\hline
\end{tabular}

Table. 1. Planets distances from the Sun and the prediction of Bode rule. Bode rule cannot predict the distance of Neptune from the Sun.

\section{Wave Function and Bode Rule}

Consider a standing and cosine wave function with a wavelength $\lambda=0.6 \mathrm{AU}$; if we assume that the first node of this wave is at a distance of $0.1 A U$ from the Sun the next nodes are at the distances of $0.4 A U, 0.7 A U, 1 A U, 1.3 A U, 1.6 A U, \ldots 2.8 A U, \ldots$ from the Sun. Each node is $0.3 A U$ ahead of the previous node. If we consider the planets of solar system in the position of the nodes of this wave, in such a case, there is no planet on the first node ( $0.1 A U)$ and Mercury is on the second node, Venus is on the third node, Earth is on the fourth node, Mars is on the sixth node, and the position of fifth node (1.3 $A U)$ is empty. The seventh, eighth, and ninth nodes are empty, and Ceres is on the tenth node. Jupiter is placed on the eighteenth node and Saturn is on the thirtythird node, and Uranus, Neptune, and Pluto are on the nodes farther from the Sun. As you can see, a wave function, with the wavelength $\lambda=0.6 \mathrm{AU}$, easily predicts the position of the planets and it seems that a huge and standing wave plays a role in determining the position of the planets in solar system. Therefore, we can consider the reason for the Bode rule to be the existence of a large cosine wave in solar system that oscillates along the axis perpendicular to the plane of solar system. In this article I will obtain the equation of this wave function. But what does this wave belong to? I answer this question in this article. The presence of a huge cosine wave in solar system seems strange at first glance, but quantum mechanics eradicates our surprise. Based on quantum mechanics, a wave packet can be assigned to each object called the 'associated matter wave 'of that object, and this associated wave is the solution of the Schrodinger equation. In this article, I prove that the above standing and cosine wave function (the wave function with the wavelength $\lambda=0.6 \mathrm{AU}$ ) is the solution of the Schrodinger equation and so, based on Quantum mechanics, this wave must belong to an object; I demonstrate that this object is the sun. It is sun that has created a standing wave around itself and provided a definite orbit for the planets ${ }^{1}$.

\footnotetext{
${ }^{1}$ I know that Quantum mechanics, the Schrodinger equation, and the de Broglie wavelength relation can only be used for subatomic scale and objects. But, in this article, I prove that quantum mechanics is also valid in astronomical scale.
} 
As mentioned, a cosine and standing wave function, with the wavelength $\lambda=0.6 A U\left(k=\frac{2 \pi}{\lambda}=\right.$ $\left.\frac{10 \pi}{3}\right)$, can predict the position of the planets in solar system. First I want to derive the phase constant of this wave function. Any wave in which the variables $x$ and $t$ are entered as a combination of $k x \pm w t$ is a traveling wave [2]. For example, the equation $\sin (k x-w t+\emptyset)$ shows a traveling wave. The zeros of this sine function, which are correspond to the nodes of this wave, are found at positions $x_{n}$ as follows:

$$
\sin \left(k x_{n}-w t+\emptyset\right)=\sin (n \pi)=0 \Rightarrow 2 \pi\left(\frac{x_{n}}{\lambda}-f t+\frac{\emptyset}{2 \pi}\right)=n \pi \Rightarrow x_{n}=\frac{\lambda}{2}\left(n+2 f t-\frac{\emptyset}{\pi}\right)
$$

Where $f$ is frequency. As you can see, by variation $t$, the location of nodes $\left(x_{n}\right)$ is changed. Therefore, the form of a standing wave must be either $\cos (w t) \cos (k x+\emptyset)$ or $\sin (w t) \cos (k x+\emptyset)$. Thus, for the nodes of a standing wave we have $\cos (k x+\emptyset)=0$. As mentioned, Mercury is on the second node of the solar system wave function (the second node corresponds to the phase $\frac{3 \pi}{2}$ ). We have:

$x_{\text {Mercury }}=0.4 A U \Rightarrow \psi\left(x_{\text {Mercury }}\right)=0 \Rightarrow \cos (k x+\emptyset)=0 \Rightarrow k x+\emptyset=\frac{3 \pi}{2} \stackrel{k=\frac{10 \pi}{3}}{\Longrightarrow} \emptyset=\frac{\pi}{6}$

Having $k$ and $\emptyset$, we can easily find the position of the other planets using the equation $k x+\emptyset=\frac{\pi}{2}, \frac{3 \pi}{2}, \frac{5 \pi}{2}, \ldots ., \frac{(2 m-1) \pi}{2}$ (Where $\mathrm{m}$ is the node number). For example

or

$$
k x_{\text {Venus }}+\emptyset=\frac{5 \pi}{2} \Rightarrow \frac{10 \pi}{3} x_{\text {Venus }}+\frac{\pi}{6}=\frac{5 \pi}{2} \Rightarrow x_{\text {Venus }}=0.7 \mathrm{AU}
$$

80

1

$$
\begin{aligned}
& k x_{\text {Earth }}+\emptyset=\frac{7 \pi}{2} \Rightarrow \frac{10 \pi}{3} x_{E a r t h}+\frac{\pi}{6}=\frac{7 \pi}{2} \Rightarrow x_{\text {Earth }}=1 \mathrm{AU} \\
& k x_{\text {Mars }}+\emptyset=\frac{11 \pi}{2} \Rightarrow \frac{10 \pi}{3} x_{\text {Mars }}+\frac{\pi}{6}=\frac{11 \pi}{2} \Rightarrow x_{\text {Mars }}=1.6
\end{aligned}
$$

The distances of the other planets can also be calculated in the same way, which is quite consistent with experience. According to the above equation $\left(k x+\emptyset=\frac{(2 m-1) \pi}{2}\right)$, Neptune is on the ninetyeighth node, which corresponds to the phase $\frac{195 \pi}{2}$. Contrary to the Bode rule, which is not able to predict the distance of Neptune, our wave theory predicts the position of Neptune. Therefore, a cosine and standing wave function with $\emptyset=\frac{\pi}{6}$ and $k=\frac{10 \pi}{3}$ can be attributed to solar system. But what is the general equation of this wave function? As mentioned, The equation of solar system must contain a component with the equation $\cos \left(\frac{10 \pi}{3} x+\frac{\pi}{6}\right)$ and on the other hand, this wave must be standing so that the position of the nodes (planets) does not change. Therefore, the form of solar system wave function must be either $\cos (\delta w t) \cos \left(\frac{10 \pi}{3} x+\frac{\pi}{6}\right)$ or $\sin (\delta w t) \cos \left(\frac{10 \pi}{3} x+\frac{\pi}{6}\right)$. Due 
91 to the symmetry of solar system around the sun we choose the equation $\cos (\delta w t) \cos \left(\frac{10 \pi}{3} x+\frac{\pi}{6}\right)$ and then we will show that our choice is $\operatorname{correct}(\delta$ is a constant number that we will derive its value). Since solar system has a certain size and is not infinitely wide, its wave equation must be localized (a wave packet). If we consider an expression in the form $e^{-\gamma x^{2}}$ (which is a Gaussian function and plays the role of a wave envelope) in the final equation, in such a case, the final equation is a localized wave or a wave packet, (the value of $\gamma$, which is a positive number, will be obtained in the following) thus, the primary form of the wave function of solar system is as follows (equation 1) and the planets are on the nodes of this wave function (Fig. 1):

$$
\begin{cases}\psi(x, t)=C \cos (\delta w t) \cos \left(\frac{10 \pi}{3} x+\frac{\pi}{6}\right) e^{-\gamma x^{2}} & x \geq 0 \\ \psi(x, t)=C \cos (\delta w t) \cos \left(\frac{10 \pi}{3} x-\frac{\pi}{6}\right) e^{-\gamma x^{2}} & x \leq 0\end{cases}
$$

In equation $1, \gamma, \mathrm{C}$ and $\delta$ are constant values and we obtain their values in this article.

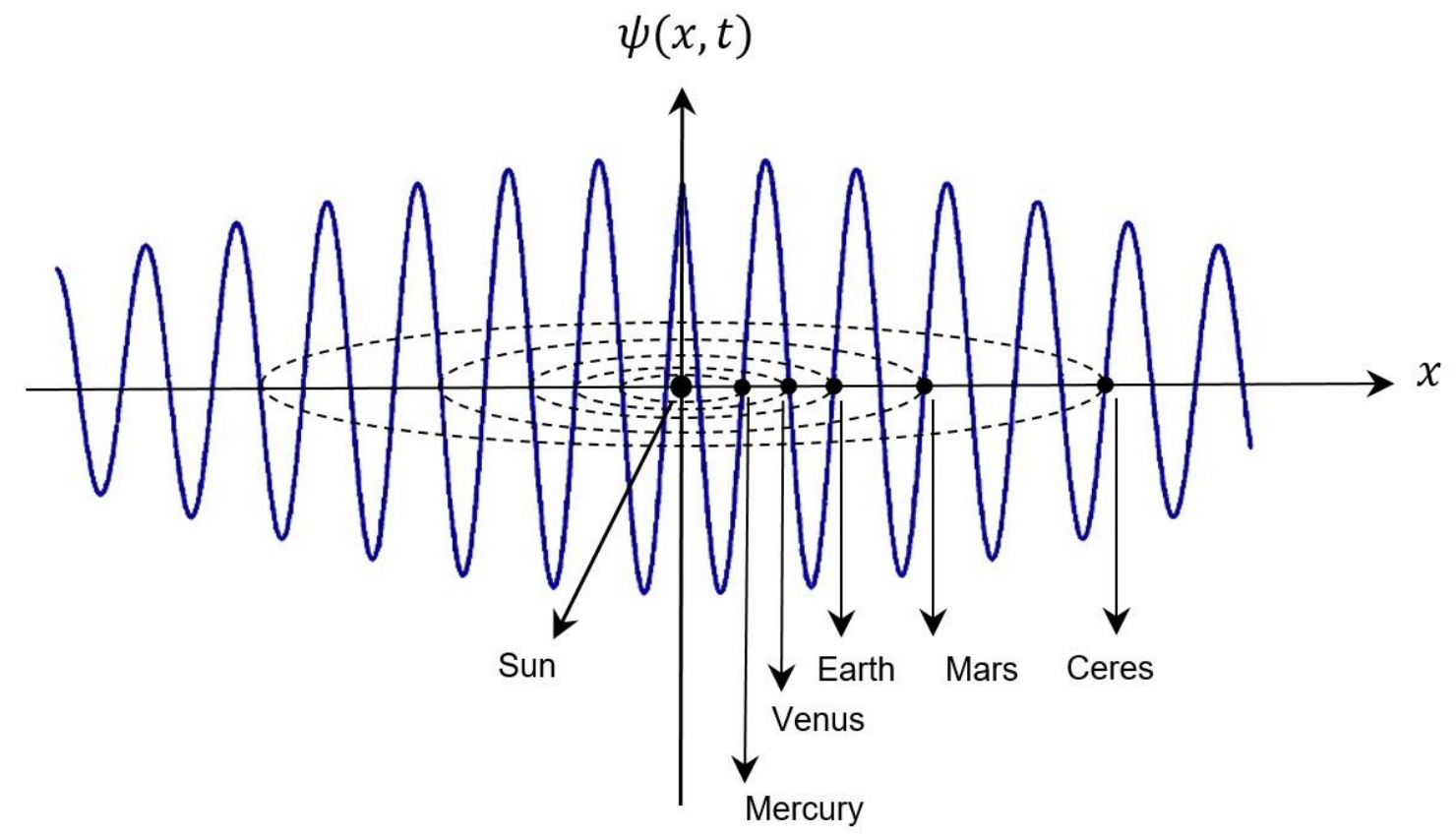

Fig. 1. Solar system standing wave packet with $\lambda=0.6 \boldsymbol{A U}$ and $\emptyset=\frac{\pi}{6}$. Diagram of $\psi(x, t)$ at the moment $\mathrm{t}=0$. The value of $\psi(0,0)$ is equal to $\sqrt{3} C / 2$. This diagram is drawn by a certain value of $C, \delta$ and $\gamma$ in equation 1 , which we will obtain their value at the end of the article. As you can see, the planets are on the nodes of the wave function. Jupiter, Saturn, Uranus, Neptune, and Pluto are on the nodes farther from the Sun. In Fig. 1, the wave oscillates along the $\psi$ axis over time. But the nodes and the anti-nodes do not move relative to each other along the $\mathrm{x}$-axis. This does not mean that the wave packet is stationary in the space; it is just like passengers sitting on a train who do not move relative to each other but 109 the train is moving relative to the rails. In the same way, solar system wave packet (equation 1) is 
a standing wave that rotates, along with solar system, around the center of the galaxy. As you see, equation (1) can easily predict the position of planets. This equation is the "wave equation of solar system". In the continuation of the article, we will prove that this equation is the Real part of a solution of Schrodinger equation ${ }^{2}$ and that's why we can assign it to an object like the sun.

\section{Associated Wave Packet of Sun}

In quantum mechanics, in order to build a wave packet from a set of infinite number of flat matter waves $A_{0} e^{i(k x-w t)}$ that move in one direction, we sum them together based on the superposition principle [3],[4]. In this case, the net wave is a wave packet, and this wave packet is the solution of the Schrodinger equation. But this wave packet is a traveling wave packet, and now we want to see if it is possible to make a standing wave packet from the sum of the infinite number of flat matter waves. To obtain this standing wave packet, we proceed as follows.

Schrodinger wave equation for a free particle in one dimension is as follows:

$$
i \hbar \frac{\partial}{\partial t} \psi(x, t)=-\frac{\hbar^{2}}{2 m} \Delta \psi(x, t)
$$

Where $\Delta$ is the Laplace operator. This equation clearly has the following solutions [3].

$$
\psi(x, t)=\mathrm{A}(x, t)=A_{0} e^{i(k x \pm w t \pm \emptyset)}=A_{0} e^{i(k x \pm \emptyset)} e^{ \pm i w t}
$$

On the condition that $k$ and $w$ satisfy the relation below:

$$
w=\frac{\hbar k^{2}}{2 m}
$$

$A_{0} e^{i(k x-w t \pm \emptyset)}$ represents the matter wave propagating in the positive direction of the $\mathrm{x}$-axis ${ }^{3}$, and $A_{0} e^{i(k x+w t \pm \varnothing)}$ represents the matter wave propagating in the negative direction of $\mathrm{x}$-axis. Equation 4 is obtained by combining the Planck equation $E=\hbar w$ and the de Broglie equation $p=\hbar k$.

The flat wave with equation 3 represents an associated wave with a matter particle whose probability of presence is uniform throughout all space ${ }^{4}$. But real matter particles are not extensive throughout space, they are localized, and in quantum we are seeking the description of localized matter particles. So we have to use the equation of a wave packet instead of equation 3 . The equation of a matter wave packet is obtained from the superposition of (sum of) the waves of Equation 3. And we know from the principle of superposition that this net wave is the solution of the Schrodinger equation (Equation 2).

In order to obtain an equation for a 'standing wave packet ', we must calculate the superposition of a set of infinite numbers of flat matter waves which move in opposite directions to each other. The net wave is a standing wave packet, which is the solution of the Schrodinger equation.

\footnotetext{
${ }^{2}$ As you know, the solutions of the Schrodinger equation have two parts: Real and Imaginary.

${ }^{3}$ The sign \pm behind $\emptyset$ means that both $A_{0} e^{i(k x-w t-\varnothing)}$ and $A_{0} e^{i(k x-w t+\emptyset)}$ are the solution of the Schrodinger equation.

${ }^{4}$ According to the uncertainty principle $\Delta x . \Delta p \geq \hbar$ and the formula $p=\hbar k$, when $\mathrm{k}$ has a certain value then we have $\Delta p=0$ and therefore, the value of $\Delta x$ is infinite.
} 
First we calculate the superposition of the waves moving in the positive direction of the $\mathrm{x}$-axis and add this net wave to the superposition of the waves moving in the negative direction of the $\mathrm{x}$-axis. Consider a set of infinite numbers of flat matter waves $A_{0} e^{i(k x-w t+\varnothing)}$, which move in the positive direction of the $\mathrm{x}$-axis, and suppose that the angular frequency of these waves is equal to $w_{0}$ and the wave number of these waves is around the median of $k_{0}$ and between $k_{0}+\Delta k / 2$ and $k_{0}-\Delta k / 2^{5}$. In such a case, the resultant of these waves, using the superposition principle, is a wave packet with equation 5 [3][4].

$$
\psi_{\text {total }}(x, t)=A_{\text {total }}(x, t)=\int A_{0}(k) e^{i\left(k x-w_{0} t+\varnothing\right)} d k
$$

Where k means $k_{x}$. In equation $5, A_{0}(k)$ is a Gaussian and symmetric function.

$$
A_{0}(k)=e^{-\alpha\left(k-k_{0}\right)^{2}}
$$

In equation $6, \alpha$ is a constant with a positive value and shows the width of the bell-shaped function of $A_{0}(k)$. Since equation 5 is derived from the superposition principle, it is the solution of the Schrodinger equation.

To obtain $\psi_{\text {total }}(x, t)$ from equation 5 , we calculate the superposition of all of the waves in one moment, which we consider to be the origin of time $(t=0)$, and then we can obtain the net wave at any other time. We have:

$$
\psi(x, 0)=\int A_{0}(k) e^{i(k x+\varnothing)} d k
$$

The above equation is the momentary image of the net wave. Multiply equation 7 by $e^{i k_{0} x-i k_{0} x}$. We have:

$$
\psi(x, 0)=e^{i\left(k_{0} x+\emptyset\right)} \int A_{0}(k) e^{i\left(k-k_{0}\right) x} d k
$$

Considering $k^{\prime}=k-k_{0}$, we have:

$$
\psi(x, 0)=e^{i\left(k_{0} x+\emptyset\right)} \int e^{-\alpha k^{2}} e^{i k^{\prime} x} d k^{\prime}
$$

Using the variable transformation $k^{\prime}-\frac{i x}{2 \alpha}=q$ (this variable transformation can be explained based on the theory of complex variables [4]) and the Gaussian integral $\int_{-\infty}^{\infty} d q e^{-\alpha q^{2}}=\sqrt{\frac{\pi}{\alpha}}$, equation 9 can be calculated. After placement and simplification, we reach the following final solution [4]:

\footnotetext{
${ }^{5}$ In the Electromagnetic waves we cannot consider one $w_{0}$ for two or many waves in which their $k$ is different from each other, because for all of the EM waves we have: $w=c k$ where $c$ is the speed of light. But for matter waves the issue is different. In the matter waves we have $w=\frac{\hbar k^{2}}{2 m}$. As you can see $w$ is the function of $k$ and $m$. Therefore, it is possible to choose one value of $w_{0}$ for the waves in which their $k$ is different from each other.
} 


$$
\psi(x, 0)=\sqrt{\frac{\pi}{\alpha}} e^{i\left(k_{0} x+\varnothing\right)} e^{-\frac{x^{2}}{4 \alpha}}
$$

This equation shows us the form of a traveling wave packet at a point in time. But how are the time variations of Equation 10? As mentioned, we consider $w$ for each wave is equal to $w_{0}$, We have:

$$
\psi(x, t)=\int A_{0}(k) e^{i\left(k x-w_{0} t+\emptyset\right)} d k=\int e^{-\alpha k^{2}} e^{i\left(k x-w_{0} t+\varnothing\right)} d k
$$

We put $e^{i k_{0} x-i k_{0} x}$ in the equation:

$$
\psi(x, t)=e^{i\left(k_{0} x+\emptyset\right)-i w_{0} t} \int e^{-\alpha k^{2}} e^{i k^{\prime} x} d k^{\prime}
$$

This integral is similar to integral 9, which led to $\psi(x)$ (Equation 10). Therefore, the output wave equation of a set of the infinite number of waves $A_{0} e^{i\left(k x-w_{0} t+\varnothing\right)}$, which are moving in the positive direction of the $x$-axis, is equal to [4];

$$
\begin{gathered}
\psi(x, t)=\psi_{1}(x, t)=\sqrt{\frac{\pi}{\alpha}} e^{i\left(k_{0} x+\emptyset-w_{0} t\right)} e^{-\frac{x^{2}}{4 \alpha}} \\
e^{i \theta}=\cos \theta+i \sin \theta \Rightarrow \operatorname{Re} \psi_{1}(x, t)=\sqrt{\frac{\pi}{\alpha}} \cos \left(k_{0} x-w_{0} t+\emptyset\right) e^{-\frac{x^{2}}{4 \alpha}}
\end{gathered}
$$

Due to the presence of the factor $k_{0} x-w_{0} t$, Equation 12 represents a traveling wave packet that propagates in the positive direction of the $x$-axis [2]. This means that the location of the nodes is not known.

Similarly, we use the recent trend to obtain the superposition of flat waves traveling in the negative direction of the $x$-axis, i.e. $A_{0} e^{i\left(k x+w_{0} t+\varnothing\right)}$. If we do this, we get to equation 13:

$$
\begin{aligned}
& \psi_{2}(x, t)=\sqrt{\frac{\pi}{\alpha}} e^{i\left(k_{0} x+\emptyset+w_{0} t\right)} e^{-\frac{x^{2}}{4 \alpha}} \\
& \operatorname{Re} \psi_{2}(x, t)=\sqrt{\frac{\pi}{\alpha}} \cos \left(k_{0} x+w_{0} t+\emptyset\right) e^{-\frac{x^{2}}{4 \alpha}}
\end{aligned}
$$

This equation shows a traveling wave packet that propagates in the negative direction of the $x$-axis.

Now we sum up the two equations 14 and 12 together to get the final wave.

$$
\operatorname{Re} \psi_{\text {total }}(x, t)=\operatorname{Re} \psi_{1}+\operatorname{Re} \psi_{2}
$$

Thus:

$$
\operatorname{Re} \psi_{\text {total }}(x, t)=\sqrt{\frac{\pi}{\alpha}} e^{-\frac{x^{2}}{4 \alpha}}\left[\cos \left(k_{0} x-w_{0} t+\emptyset\right)+\cos \left(k_{0} x+w_{0} t+\emptyset\right)\right]
$$


Using the equation $\cos \alpha+\cos \beta=2 \cos \frac{1}{2}(\alpha+\beta) \cos \frac{1}{2}(\alpha-\beta)$ and $\cos (\theta)=\cos (-\theta)$ we obtain the equation of a standing wave packet.

$$
\left\{\begin{array}{l}
\alpha=k_{0} x-w_{0} t+\emptyset \\
\beta=k_{0} x+w_{0} t+\emptyset
\end{array} \Rightarrow \operatorname{Re} \psi_{\text {total }}(x, t)=2 \sqrt{\frac{\pi}{\alpha}} \cos \left(k_{0} x+\emptyset\right) \cos \left(w_{0} t\right) e^{-\frac{x^{2}}{4 \alpha}}\right.
$$

There is not the structure of $k x \pm w t$ in Equation 16 so the $\psi_{\text {total }}$ is a standing wave. As you observe, Equation 16, which is the real part of a solution of the Schrodinger equation, is exactly the same as equation 1 for $x \geq 0$, which is solar system wave function. It means that equation 1 is the real part of a solution of the Schrodinger equation. It means that the Schrodinger equation and quantum mechanics are valid in astronomical scale. This is a great achievement in physics. by comparing equation 16 and equation 1 , we have

$$
\delta=1, \gamma=\frac{1}{4 \alpha} \quad \text { and } \quad C=2 \sqrt{\frac{\pi}{\alpha}}
$$

If we put these values in Equation 1, then we get the final equation of solar system wave function for $x \geq 0$ :

$$
\operatorname{Re} \psi_{t}(x, t)=2 \sqrt{\frac{\pi}{\alpha}} \cos \left(w_{0} t\right) \cos \left(\frac{10 \pi}{3} x+\frac{\pi}{6}\right) e^{-\frac{x^{2}}{4 \alpha}} \quad x \geq 0
$$

Equation 17 is obtained by calculating the superposition of a set of infinite number of waves of $A_{0} e^{i\left(k x-w_{0} t+\emptyset\right)}$ and $A_{0} e^{i\left(k x+w_{0} t+\emptyset\right)}$ that move in opposite directions to each other (pay attention to the + sign behind $\varnothing$ ). Now if we sum a set of infinite number of flat wave functions with the equations $A_{0} e^{i\left(k x-w_{0} t-\emptyset\right)}$ and $A_{0} e^{i\left(k x+w_{0} t-\varnothing\right)}$ (pay attention to the - sign behind $\emptyset$ ) together, by following the path we have taken from equation 5 to equation 17, we reach the following relation;

$$
\operatorname{Re} \psi_{t}(x, t)=2 \sqrt{\frac{\pi}{\alpha}} \cos \left(w_{0} t\right) \cos \left(\frac{10 \pi}{3} x-\frac{\pi}{6}\right) e^{-\frac{x^{2}}{4 \alpha}}
$$

Which is in the form of equation 1 for $x \leq 0$. Therefore, the final form of solar system wave function (Equation 1) is as follows:

$$
\begin{cases}\operatorname{Re} \psi(x, t)=2 \sqrt{\frac{\pi}{\alpha}} \cos \left(w_{0} t\right) \cos \left(\frac{10 \pi}{3} x+\frac{\pi}{6}\right) e^{-\frac{x^{2}}{4 \alpha}} & x \geq 0 \\ \operatorname{Re} \psi(x, t)=2 \sqrt{\frac{\pi}{\alpha}} \cos \left(w_{0} t\right) \cos \left(\frac{10 \pi}{3} x-\frac{\pi}{6}\right) e^{-\frac{x^{2}}{4 \alpha}} & x \leq 0\end{cases}
$$

In this equation, the larger the $\alpha$, the more the width of wave packet is, along the $\mathrm{x}$-axis. We drew Fig. 1 by $\alpha=6$.

Here we demonstrated that Solar system wave equation (equation 1 or 18) is the real part of a solution of the Schrodinger equation. So we can assign it to an object in Solar system. The closest star to solar system is at a distance of 4.8 light-years, which is so far. And the biggest and heavyset object in solar system is Sun. Therefore, the wave function of solar system can only belong to the sun. But, according to the very large mass of the sun, using the de Broglie equation $\left(\lambda=\frac{h}{m v}\right)$ the 
wavelength of $0.6 \mathrm{AU}$ will not obtain. So instead of Planck constant we have to choose another value for celestial objects. We call this new value the Planck constant in Astronomy $\left(h_{\text {Astronomy }}\right)$ abbreviated as $h_{A}$ and we have: $\lambda_{A}=\frac{h_{A}}{P}$. Based on a clever conjecture in his 1924 dissertation, de Broglie hypothesized that the equation $\lambda=\frac{h}{P}$, which is true for photons, could also be applied to matter particles [5]. Here, too, we assume that there is a relation in the form of $\lambda_{A}=\frac{h_{A}}{P}$ on the scale of astronomy. In such a case, the Schrodinger equation on the astronomical scale can be written as follows:

$$
i \hbar_{A} \frac{\partial}{\partial t} \psi(x, t)=-\frac{\hbar_{A}^{2}}{2 m} \Delta \psi(x, t)
$$

If we follow the path of proving the Schrodinger wave equation and put the value $\hbar_{A}$ instead of $\hbar$, we reach equation 19. The Davisson-Germer experiment [6] is considered as the confirmation of de Broglie equation and the Schrodinger equation at the atomic scale, and the Regularity of the distances of the planets from the sun can be considered as the confirmation of equation 19 and the formula $\lambda_{A}=\frac{h_{A}}{P}$. Moreover, for the celestial wave packet we have $w=\frac{\hbar_{A} k^{2}}{2 m}$.

\section{Conclusion}

In this article, by investigating the distances of the planets of solar system from the sun, we concluded that a huge, cosine, and standing wave packet surrounds our solar system. Using Mathematics calculations, it was proved that this wave packet belongs to the sun. It is the sun that has created a standing wave around itself and provided a definite orbit for the planets. Finally, I obtained the Schrodinger equation in astronomy, which describes the behavior of huge astronomical wave packets. My wave theory predicts the distances of all of the planets (including Neptune), plus Ceres and Pluto, but as we have seen, the Bode rule does not predict the distance of Neptune. Describing the distances of the planets by a quantum wave packet can be considered as another confirmation of the validity of quantum theory. It seems that, we cannot present any justification for Bode rule except wave theory.

In addition to the astronomical scale, in the atomic scale, according to this article, it is very likely that electrons are located on the nodes of the associated wave packet of the atom nucleus. This issue can be discussed and investigated in future studies.

\section{References:}

[1]. Carrol, B. Ostlie, D. An Introduction to Modern Astrophysics (Cambridge University Press, ed. 2, 2017), pp. 716 717

[2]. Walker, J. Halliday, D. Fundamentals of Physics. (Wiley Ltd, ed. 10, 2007), pp. 449-450

[3]. Cohen-tannoudji, C. Diu, B. Quantum Mechanics. (John Wiley \& Sons Inc, Vol. 1, 1977), pp. 21-22

[4]. Gasiorowicz, S. Quantum Physics (John Wiley \& Sons Inc, ed. 1, 1974), pp. 27-31

[5]. De Broglie, L. On the Theory of Quanta. Ann. de Phys, 10th serie, Vol. III (1925).

[6]. Davisson, C. Germer, L. Reflection of Electrons by a Crystal of Nickel. Proceedings of the National Academy of Sciences of the United States of America. 14 (4). (1928), pp. 317-322. 